\title{
Exact Phase Transitions in Random Constraint Satisfaction Problems
}

$\mathrm{Ke} \mathrm{Xu}$

Wei Li

National Laboratory of Software Development Environment, Department of Computer Science and Engineering, Beijing University of Aeronautics and Astronautics, Beijing, 100083, P.R. China
KEXU@NLSDE.BUAA.EDU.CN

LIWEI@NLSDE.BUAA.EDU.CN

\begin{abstract}
In this paper we propose a new type of random CSP model, called Model RB, which is a revision to the standard Model B. It is proved that phase transitions from a region where almost all problems are satisfiable to a region where almost all problems are unsatisfiable do exist for Model RB as the number of variables approaches infinity. Moreover, the critical values at which the phase transitions occur are also known exactly. By relating the hardness of Model RB to Model B, it is shown that there exist a lot of hard instances in Model RB.
\end{abstract}

\section{Introduction}

Since the seminal paper of Cheeseman, Kanefsky and Taylor (1991) appeared, there has been a great amount of interest in the study of phase transitions in NP-complete problems. However, it seems to be very difficult to prove the existence of this phenomenon or to obtain the exact location of the transition points for such problems. For example, in random 3$\mathrm{SAT}$, it is known from experiments that the phase transition will occur when the ratio of clauses to variables is approximately 4.3 (Mitchell, Selman, \& Levesque, 1992). Another experimental estimate of the transition point suggested by Kirkpatrick and Selman (1994) is 4.17. They used finite-size scaling methods from statistical physics to derive the result. In contrast with the experimental studies, the theoretical work has only given some loose but hard won bounds on the location of the transition point. Currently, the best known lower bound and upper bound are 3.003 (Frieze \& Suen, 1996) and 4.602 (Kirousis et al., 1998) respectively. Recently, Friedgut (1999) made tremendous progress towards establishing the existence of a threshold for random $k$-SAT by proving that the width of the transition region narrows as the number of variables increases. But we still can not obtain the exact location of the phase transition point from this approach.

In fact, SAT is a special case of the constraint satisfaction problem (CSP). CSP has not only important theoretical value in artificial intelligence, but also many immediate applications in areas ranging from vision, language comprehension to scheduling and diagnosis (Dechter, 1998). In general, CSP tasks are computationally intractable (NP-hard) (Dechter, 1998). In recent years random constraint satisfaction problems have also received great attention, both from an experimental and a theoretical point of view (Achlioptas et al., 1999; Cheeseman et al., 1991; Frost \& Dechter, 1994; Gent et al., 1999; Hogg, 1996; Larrosa \& Meseguer, 1996; Prosser, 1996; Purdom, 1997; Smith \& Dyer, 1996; Smith, 1999; Williams 
\& Hogg, 1994). Williams and Hogg (1994) developed a technique to predict where the hardest problems are to be found and where the fluctuations in difficulty are greatest in a space of problem instances. They have also shown that their predictions of the critical value agree well with the experimental data. Smith and Dyer (1996) studied the location of the phase transition in binary constraint satisfaction problems and discussed the accuracy of a prediction based on the expected number of solutions. Their results show that the variance of the number of solutions can be used to set bounds on the phase transition and to indicate the accuracy of the prediction. Recently, a theoretical result by Achlioptas et al. (1999) shows that many models commonly used for generating random CSP instances do not have an asymptotic threshold due to the presence of flawed variables. More recently, Gent et al. (1999) have shown how to introduce structure into the conflict matrix to eliminate flaws.

In this paper we propose a new type of random CSP model, called Model RB, which is a revision to the standard Model B (Gent et al., 1999; Smith \& Dyer, 1996). It is proved that the phase transition phenomenon does exist for Model RB as the number of variables approaches infinity. More precisely, there exist two control parameters $r, p$ and the corresponding critical values $r_{c r}, p_{c r}$ such that for each fixed value $r<r_{c r}$ or $p<p_{c r}$, a random CSP instance generated following Model RB is satisfiable with probability tending to 1 as the number of variables approaches infinity, and when $r>r_{c r}$ or $p>p_{c r}$, unsatisfiable with probability tending to 1 . Moreover, the critical values $r_{c r}$ and $p_{c r}$ are also known exactly. By relating the hardness of Model RB to Model B, it is shown that Model RB actually has a lot of hard instances.

\section{Definitions and Notations}

A constraint satisfaction problem (CSP) consists of a finite set $U=\left\{u_{1}, \cdots, u_{n}\right\}$ of $n$ variables and a set of constraints. For each variable $u_{i}$, a domain $D_{i}$ with $d_{i}$ elements is specified; a variable can only be assigned a value from its domain. For $2 \leq k \leq n$ a constraint $C_{i 1, i 2, \cdots, i k}$ consists of a subset $\left\{u_{i 1}, u_{i 2}, \cdots, u_{i k}\right\}$ of $U$ and a relation $R_{i 1, i 2, \cdots, i k} \subseteq$ $D_{i 1} \times \cdots \times D_{i k}$, where $i 1, i 2, \cdots, i k$ are distinct. $C_{i 1, i 2, \cdots, i k}$ is called a $k$-ary constraint which bounds the variables $u_{i 1}, \cdots, u_{i k}$. $R_{i 1, i 2, \cdots, i k}$ specifies all the allowed tuples of values for the variables $u_{i 1}, \cdots, u_{i k}$ which are compatible with each other. A solution to a CSP is an assignment of a value to each variable from its domain such that all the constraints are satisfied. A constraint $C_{i 1, i 2, \cdots, i k}$ is satisfied if the tuple of values assigned to the variables $u_{i 1}, \cdots, u_{i k}$ is in the relation $R_{i 1, i 2, \cdots, i k}$. A CSP that has a solution is called satisfiable; otherwise it is unsatisfiable. In this paper, the probability of a random CSP instance being satisfiable is denoted by $\operatorname{Pr}($ Sat $)$.

We assume that $k \geq 2$ and all the variable domains contain the same number of values $d=n^{\alpha}$ in Model RB (where $\alpha$ is a constant). The generation of a random CSP instance in Model RB is done in the following two steps:

Step 1. We select with repetition $t=r n \ln n$ random constraints. Each random constraint is formed by selecting without repetition $k$ of $n$ variables.

Step 2. For each constraint we uniformly select without repetition $q=p \cdot d^{k}$ incompatible tuples of values, i.e., each constraint relation contains exactly $(1-p) \cdot d^{k}$ compatible tuples of values. 
The parameter $r$ determines how many constraints are in a CSP instance, while $p$ determines how restrictive the constraints are.

The following definitions will be needed in section 4 when we derive the expectation of the second moment of the number of solutions.

Definition 1 An assignment pair is an ordered pair $\left\langle t_{i}, t_{j}\right\rangle$ of assignments to the variables in $U$, where $t_{i}=\left(a_{i 1}, a_{i 2}, \cdots, a_{i n}\right)$ and $t_{j}=\left(a_{j 1}, a_{j 2}, \cdots, a_{j n}\right)$ with $a_{i l}, a_{j l} \in D_{l}$. An assignment pair $\left\langle t_{i}, t_{j}\right\rangle$ satisfies a CSP if and only if both $t_{i}$ and $t_{j}$ satisfy this CSP. The set that consists of all the assignment pairs is denoted by $A_{\text {pair }}$.

Definition 2 Similarity number $S^{f}: A_{\text {pair }} \mapsto\{0,1,2, \cdots\}$,

$$
S^{f}\left(\left\langle t_{i}, t_{j}\right\rangle\right)=\sum_{l=1}^{n} \operatorname{Sam}\left(a_{i l}, a_{j l}\right)
$$

where the function Sam is defined as follows:

$$
\operatorname{Sam}\left(a_{i l}, a_{j l}\right)= \begin{cases}1 & \text { if } a_{i l}=a_{j l} \\ 0 & \text { if } a_{i l} \neq a_{j l}\end{cases}
$$

The similarity number of an assignment pair is equal to the number of variables at which the two assignments of this assignment pair take the identical values. By Definition 2 it is easy to see that $0 \leq S^{f}\left(\left\langle t_{i}, t_{j}\right\rangle\right) \leq n$.

\section{Main Results}

In this paper, the following theorems are proved.

Theorem 1 Let $r_{c r}=-\frac{\alpha}{\ln (1-p)}$. If $\alpha>\frac{1}{k}, 0<p<1$ are two constants and $k, p$ satisfy the inequality $k \geq \frac{1}{1-p}$, then

$$
\begin{aligned}
& \lim _{n \rightarrow \infty} \operatorname{Pr}(\text { Sat })=1 \text { when } r<r_{c r} \\
& \lim _{n \rightarrow \infty} \operatorname{Pr}(\text { Sat })=0 \text { when } r>r_{c r}
\end{aligned}
$$

Theorem 2 Let $p_{c r}=1-e^{-\frac{\alpha}{r}}$. If $\alpha>\frac{1}{k}, r>0$ are two constants and $k, \alpha$ and $r$ satisfy the inequality $k e^{-\frac{\alpha}{r}} \geq 1$, then

$$
\begin{aligned}
& \lim _{n \rightarrow \infty} \operatorname{Pr}(\text { Sat })=1 \text { when } p<p_{c r} \\
& \lim _{n \rightarrow \infty} \operatorname{Pr}(\text { Sat })=0 \text { when } p>p_{c r}
\end{aligned}
$$

\section{Proof of Theorem 1 and Theorem 2}

The expected number of solutions $E(N)$ for model RB is given by

$$
E(N)=d^{n}(1-p)^{r n \ln n}=n^{\alpha n}(1-p)^{r n \ln n}
$$

By the Markov inequality $\operatorname{Pr}(S a t) \leq E(N)$ it is not hard to show that $\lim _{n \rightarrow \infty} \operatorname{Pr}(\operatorname{Sat})=0$ when $r>r_{c r}$ or $p>p_{c r}$. Hence relations (4), (6) are proved. It is also easy to see that 
$E(N)$ is eqal to 1 when $r=r_{c r}$ or $p=p_{c r}$, and $E(N)$ grows exponentially with $n$ when $r<r_{c r}$ or $p<p_{c r}$.

The key point in the proof of relations $(3),(5)$ is to derive the expectation of the second moment $E\left(N^{2}\right)$ and give an asymptotic estimate of it. Let $\phi$ be a random CSP instance generated following Model RB. $P\left(\left\langle t_{i}, t_{j}\right\rangle\right)$ stands for the probability of $\left\langle t_{i}, t_{j}\right\rangle$ satisfying $\phi$. Now we start to derive the expression of $P\left(\left\langle t_{i}, t_{j}\right\rangle\right)$. Since each constraint is generated independently, we only need to consider the probability of $\left\langle t_{i}, t_{j}\right\rangle$ satisfying a random constraint. Assuming that the similarity number of $\left\langle t_{i}, t_{j}\right\rangle$ is equal to $S$, we have the following two cases:

(1) Each variable of a constraint is assigned the same value in $t_{i}$ as that in $t_{j}$. In this case, the probability of $\left\langle t_{i}, t_{j}\right\rangle$ satisfying the constraint is $\left(\begin{array}{c}d^{k}-1 \\ q\end{array}\right) /\left(\begin{array}{c}d^{k} \\ q\end{array}\right)$.

(2) Otherwise, the probability of $\left\langle t_{i}, t_{j}\right\rangle$ satisfying a constraint is $\left(\begin{array}{c}d^{k}-2 \\ q\end{array}\right) /\left(\begin{array}{c}d^{k} \\ q\end{array}\right)$.

The probability that a random constraint falls into the first case is $\left(\begin{array}{c}S \\ k\end{array}\right) /\left(\begin{array}{c}n \\ k\end{array}\right)$. Hence the probability into the second case is $1-\left(\begin{array}{c}S \\ k\end{array}\right) /\left(\begin{array}{c}n \\ k\end{array}\right)$. Thus we get

$$
P\left(\left\langle t_{i}, t_{j}\right\rangle\right)=\left(\frac{\left(\begin{array}{c}
d^{k}-1 \\
q
\end{array}\right)}{\left(\begin{array}{c}
d^{k} \\
q
\end{array}\right)} \cdot \frac{\left(\begin{array}{l}
S \\
k
\end{array}\right)}{\left(\begin{array}{c}
n \\
k
\end{array}\right)}+\frac{\left(\begin{array}{c}
d^{k}-2 \\
q
\end{array}\right)}{\left(\begin{array}{c}
d^{k} \\
q
\end{array}\right)} \cdot\left(1-\frac{\left(\begin{array}{c}
S \\
k
\end{array}\right)}{\left(\begin{array}{c}
n \\
k
\end{array}\right)}\right)\right)^{r n \ln n}
$$

Let $A_{S}$ be the set of assignment pairs whose similarity number is equal to $S$. It is easy to show that the cardinality of $A_{S}$ is given by

$$
\left|A_{S}\right|=d^{n}\left(\begin{array}{c}
n \\
S
\end{array}\right)(d-1)^{n-S}
$$

From the definition of $E\left(N^{2}\right)$, we have

$$
\begin{aligned}
E\left(N^{2}\right) & =\sum_{S=0}^{n}\left|A_{S}\right| P\left(t_{i}, t_{j}\right) \\
& =d^{n}\left(\begin{array}{c}
n \\
S
\end{array}\right)(d-1)^{n-S}\left(\frac{\left(\begin{array}{c}
d^{k}-1 \\
q
\end{array}\right)}{\left(\begin{array}{c}
d^{k} \\
q
\end{array}\right)} \cdot \frac{\left(\begin{array}{c}
S \\
k
\end{array}\right)}{\left(\begin{array}{c}
n \\
k
\end{array}\right)}+\frac{\left(\begin{array}{c}
d^{k}-2 \\
q
\end{array}\right)}{\left(\begin{array}{c}
d^{k} \\
q
\end{array}\right)} \cdot\left(1-\frac{\left(\begin{array}{c}
S \\
k
\end{array}\right)}{\left(\begin{array}{c}
n \\
k
\end{array}\right)}\right)\right.
\end{aligned}
$$

It is very difficult to analyze the above expression directly. First, we give an asymptotic estimate of $P\left(\left\langle t_{i}, t_{j}\right\rangle\right)$. Let $s=\frac{S}{n}$. It is obvious that $0 \leq s \leq 1$. By asymptotic analysis, we get

$$
\frac{\left(\begin{array}{c}
S \\
k
\end{array}\right)}{\left(\begin{array}{c}
n \\
k
\end{array}\right)}=\frac{\frac{S}{n}\left(\frac{S}{n}-\frac{1}{n}\right)\left(\frac{S}{n}-\frac{2}{n}\right) \cdots\left(\frac{S}{n}-\frac{k-1}{n}\right)}{\left(1-\frac{1}{n}\right)\left(1-\frac{2}{n}\right) \cdots\left(1-\frac{k-1}{n}\right)}=s^{k}+\frac{g(s)}{n}+O\left(\frac{1}{n^{2}}\right)
$$


where

$$
g(s)=\frac{k(k-1)\left(s^{k}-s^{k-1}\right)}{2}
$$

and

$$
\begin{gathered}
\frac{\left(\begin{array}{c}
d^{k}-1 \\
q
\end{array}\right)}{\left(\begin{array}{c}
d^{k} \\
q
\end{array}\right)}=\frac{d^{k}-q}{d^{k}}=1-p \\
\frac{\left(\begin{array}{c}
d^{k}-2 \\
q
\end{array}\right)}{\left(\begin{array}{c}
d^{k} \\
q
\end{array}\right)}=\frac{\left(d^{k}-q\right)\left(d^{k}-q-1\right)}{d^{k}\left(d^{k}-1\right)}=(1-p)^{2}+O\left(\frac{1}{d^{k}}\right)
\end{gathered}
$$

Note that $d=n^{\alpha}$, we have

$$
P\left(\left\langle t_{i}, t_{j}\right\rangle\right)=\left[(1-p) \cdot\left(s^{k}+\frac{g(s)}{n}\right)+(1-p)^{2} \cdot\left(1-s^{k}-\frac{g(s)}{n}\right)+O\left(\frac{1}{n^{2}}\right)+O\left(\frac{1}{n^{k \alpha}}\right)\right]^{r n \ln n}
$$

By use of the condition $\alpha>\frac{1}{k}$, we get

$$
P\left(\left\langle t_{i}, t_{j}\right\rangle\right)=(1-p)^{2 r n \ln n}\left[1+\frac{p}{1-p}\left(s^{k}+\frac{g(s)}{n}\right)\right]^{r n \ln n}\left(1+O\left(\frac{1}{n}\right)\right)
$$

For every $0<s<1$ (where $s=\frac{S}{n}$ ), the asymptotic estimate of $\left|A_{S}\right|$ is

$$
\begin{aligned}
\left|A_{S}\right| & =n^{\alpha n}\left(n^{\alpha}-1\right)^{n-n s} \frac{1}{\sqrt{2 \pi n s(1-s)}} e^{n(-s \ln s-(1-s) \ln (1-s))}\left(1+O\left(\frac{1}{n}\right)\right) \\
& =n^{2 \alpha n}\left(1-\frac{1}{n^{\alpha}}\right)^{n-n s}\left(\frac{1}{n^{\alpha}}\right)^{n s} \frac{1}{\sqrt{2 \pi n s(1-s)}} e^{n(-s \ln s-(1-s) \ln (1-s))}\left(1+O\left(\frac{1}{n}\right)\right)(10)
\end{aligned}
$$

Notice that $E(N)=n^{\alpha n}(1-p)^{r n \ln n}$, we have

$$
\left|A_{S}\right| P\left(\left\langle t_{i}, t_{j}\right\rangle\right)=E^{2}(N)\left[1+\frac{p}{1-p}\left(s^{k}+\frac{g(s)}{n}\right)\right]^{r n \ln n}\left(1-\frac{1}{n^{\alpha}}\right)^{n-n s}\left(\frac{1}{n^{\alpha}}\right)^{n s}\left(\begin{array}{c}
n \\
n s
\end{array}\right)\left(1+O\left(\frac{1}{n}\right)\right)
$$

When $n$ is sufficiently large, except the first term $E^{2}(N),\left|A_{S}\right| P\left(\left\langle t_{i}, t_{j}\right\rangle\right)$ is mainly determined by the following terms:

$$
f(s)=\left[1+\frac{p}{1-p} s^{k}\right]^{r n \ln n}\left(\frac{1}{n^{\alpha}}\right)^{n s}
$$

We can rewrite it as

$$
f(s)=e^{\left[r \ln \left(1+\frac{p}{1-p} s^{k}\right)-\alpha s\right] n \ln n}
$$

Let

$$
h(s)=r \ln \left(1+\frac{p}{1-p} s^{k}\right)-\alpha s
$$

The second derivative of $h(s)$ is

$$
h^{\prime \prime}(s)=\frac{r k p s^{k-2}\left[(k-1)(1-p)-p s^{k}\right]}{\left(1-p+p s^{k}\right)^{2}}
$$


Applying the condition $k \geq \frac{1}{1-p}$ in Theorem 1 to the above equation we can easily prove that $h^{\prime \prime}(s) \geq 0$ on the interval $0 \leq s \leq 1$. For Theorem 2 , from the condition $k e^{-\frac{\alpha}{r}} \geq 1$ it follows that the inequality $k \geq \frac{1}{1-p}$ still holds when $p<p_{c r}$. It is also not hard to show that $h(0)=0$, and $h(1)=-r \ln (1-p)-\alpha<0$ when $r<r_{c r}$ or $p<p_{c r}$. Hence we can easily prove that the unique maximum point of $h(s)$ is $s=0$ when $r<r_{c r}$ or $p<p_{c r}$. Thus the terms of $0<s \leq 1$ are negligible when $r<r_{c r}$ or $p<p_{c r}$. We only need to consider those terms near $s=0$. The process can be divided into the following three cases:

Case 1: $\alpha>1$. When $S=0(s=0)$, from the definition of $g(s)$ in Equation (11) we have

$$
\left[1+\frac{p}{1-p}\left(s^{k}+\frac{g(s)}{n}\right)\right]^{r n \ln n}=1
$$

Thus by Equation (17) we get

$$
\left|A_{S}\right| P\left(\left\langle t_{i}, t_{j}\right\rangle\right) \approx E^{2}(N)\left(1-\frac{1}{n^{\alpha}}\right)^{n} \approx E^{2}(N)
$$

When $S=1\left(s=\frac{1}{n}\right)$, it also not hard to prove that

$$
\lim _{n \rightarrow \infty}\left[1+\frac{p}{1-p}\left(s^{k}+\frac{g(s)}{n}\right)\right]^{r n \ln n}=e^{0}=1
$$

Hence we obtain

$$
\left|A_{S}\right| P\left(\left\langle t_{i}, t_{j}\right\rangle\right) \approx E^{2}(N) n^{1-\alpha} \text { when } S=1
$$

Similary,

$$
\begin{gathered}
\left|A_{S}\right| P\left(\left\langle t_{i}, t_{j}\right\rangle\right) \approx E^{2}(N) \frac{n^{2(1-\alpha)}}{2 !} \text { when } S=2 \\
\left|A_{S}\right| P\left(\left\langle t_{i}, t_{j}\right\rangle\right) \approx E^{2}(N) \frac{n^{3(1-\alpha)}}{3 !} \text { when } S=3, \cdots
\end{gathered}
$$

Summing the above terms together, we obtain

$$
E\left(N^{2}\right)=\sum_{S=0}^{n}\left|A_{S}\right| P\left(\left\langle t_{i}, t_{j}\right\rangle\right) \approx E^{2}(N) e^{n^{1-\alpha}} \approx E^{2}(N)
$$

Case 2: $\alpha=1$. By use of the method in Case 1, it can be easily shown that

$$
\begin{gathered}
\left|A_{S}\right| P\left(\left\langle t_{i}, t_{j}\right\rangle\right) \approx E^{2}(N)\left(1-\frac{1}{n}\right)^{n} \approx E^{2}(N) \frac{1}{e} \text { when } S=0 \\
\left|A_{S}\right| P\left(\left\langle t_{i}, t_{j}\right\rangle\right) \approx E^{2}(N) \frac{1}{e} \text { when } S=1 \\
\left|A_{S}\right| P\left(\left\langle t_{i}, t_{j}\right\rangle\right) \approx E^{2}(N) \frac{1}{e \cdot 2 !} \text { when } S=2 \\
\left|A_{S}\right| P\left(\left\langle t_{i}, t_{j}\right\rangle\right) \approx E^{2}(N) \frac{1}{e \cdot 3 !} \text { when } S=3, \cdots
\end{gathered}
$$


Summing the above terms together, we obtain

$$
E\left(N^{2}\right)=\sum_{S=0}^{n}\left|A_{S}\right| P\left(\left\langle t_{i}, t_{j}\right\rangle\right) \approx E^{2}(N) \frac{1}{e} \cdot e=E^{2}(N)
$$

Case 3: $\frac{1}{k}<\alpha<1$. Let $S_{0}=n^{\beta}$ (where $\beta$ is a constant and satisfies $1-\alpha<\beta<1-\frac{1}{k}$ ). It is not hard to show that when $0 \leq S \leq S_{0}\left(0 \leq s \leq n^{\beta-1}<n^{-\frac{1}{k}}\right)$, the following limit holds:

$$
\lim _{n \rightarrow \infty} \frac{p}{1-p}\left(s^{k}+\frac{g(s)}{n}\right) \cdot n \ln n=0
$$

Thus when $0 \leq S \leq S_{0}$, the asymptotic estimate of the second term in the right of Equation (17) is

$$
\left[1+\frac{p}{1-p}\left(s^{k}+\frac{g(s)}{n}\right)\right]^{r n \ln n} \approx e^{0}=1 \text { when } n \rightarrow \infty
$$

So when $0 \leq S \leq S_{0}$, the asymptotic estimate of $\left|A_{S}\right| P\left(\left\langle t_{i}, t_{j}\right\rangle\right)$ is

$$
\left|A_{S}\right| P\left(\left\langle t_{i}, t_{j}\right\rangle\right) \approx E^{2}(N)\left(\begin{array}{c}
n \\
S
\end{array}\right)\left(1-\frac{1}{n^{\alpha}}\right)^{n-S}\left(\frac{1}{n^{\alpha}}\right)^{S}
$$

It should be noted that $\left(\begin{array}{c}n \\ S\end{array}\right)\left(1-\frac{1}{n^{\alpha}}\right)^{n-S}\left(\frac{1}{n^{\alpha}}\right)^{S}$ is a binomial term whose maximum point is around $S=n^{1-\alpha}$, and $S_{0}=n^{\beta}>n^{1-\alpha}$. By asymptotic analysis, we obtain

$$
\sum_{S=0}^{S_{0}}\left(\begin{array}{c}
n \\
S
\end{array}\right)\left(1-\frac{1}{n^{\alpha}}\right)^{n-S}\left(\frac{1}{n^{\alpha}}\right)^{S} \approx \sum_{S=0}^{n}\left(\begin{array}{c}
n \\
S
\end{array}\right)\left(1-\frac{1}{n^{\alpha}}\right)^{n-S}\left(\frac{1}{n^{\alpha}}\right)^{S}=1
$$

Thus we get

$$
E\left(N^{2}\right)=\sum_{S=0}^{n}\left|A_{S}\right| P\left(\left\langle t_{i}, t_{j}\right\rangle\right) \approx E^{2}(N)
$$

Combining the above three cases gives

$$
E\left(N^{2}\right) \approx E^{2}(N) \text { when } r<r_{c r} \text { or when } p<p_{c r}
$$

Hence

$$
\lim _{n \rightarrow \infty} \frac{E^{2}(N)}{E\left(N^{2}\right)}=1 \text { when } r<r_{c r} \text { or when } p<p_{c r}
$$

By the Cauchy inequality $\operatorname{Pr}(S a t) \geq \frac{E^{2}(N)}{E\left(N^{2}\right)}$ (Bollobás, 1985), it can be easily proved that $\lim _{n \rightarrow \infty} \operatorname{Pr}(S a t)=1$ when $r<r_{c r}$ or $p<p_{c r}$. Hence Theorem 1 and Theorem 2 are proved. 


\section{The Relation between Model B and Model RB}

In this section we will explain in detail how Model RB revises Model B and show the hardness of Model RB by relating it to Model B. From the previous papers (Gent et al., 1999; Smith \& Dyer, 1996) we know that the generation of a random CSP instance in the standard Model B (which is often written as $\left\langle n, d, p_{1}, p_{2}\right\rangle$ ) is done in the following two steps: Step 1. We select with repetition $t=p_{1} \frac{n(n-1)}{2}$ random constraints. Each random constraint is formed by selecting without repetition 2 of $n$ variables.

Step 2. For each constraint we uniformly select without repetition $q=p_{2} \cdot d^{2}$ incompatible tuples of values, i.e., each constraint relation contains exactly $\left(1-p_{2}\right) \cdot d^{2}$ compatible tuples of values.

Since the standard Model B is a binary CSP model, we will only consider the binary case of Model RB in this section. In the previous papers Model B was used to test the CSP algorithms in the following way. Given the values of $n, d$ and $p_{1}$, the constraint tightness $p_{2}$ was varied from 0 to 1 in steps of $\frac{1}{d^{2}}$. At each setting of $\left\langle n, d, p_{1}, p_{2}\right\rangle$ a fixed number of instances (e.g. 100) were generated. The search algorithm was then applied to each instance. Finally numerous statistics about the search cost and the probability of being satisfiable were gathered. In fact, the two steps of forming a constraint and selecting incompatible tuples of values in Model RB is exactly the same as those in Model B. The significant difference between Model B and Model RB is that Model RB restricts how fast the domain size and the number of constraints increase with the number of variables while Model B does not, which may lead to the result that many instances of Model B suffer from being asymptotically trivially insoluble (Achlioptas et al., 1999) while Model RB avoids this problem. But it is easy to see that given the values of $n, d$ and $p_{1}$, for the setting $\left\langle n, d, p_{1}, p_{2}\right\rangle$ of Model B there is an equivalent setting in Model RB with the same number of variables as that in $\left\langle n, d, p_{1}, p_{2}\right\rangle, \alpha=\frac{\ln d}{\ln n}$ and $r=\frac{p_{1}(n-1)}{2 \ln n}\left(\right.$ Let $n^{\alpha}=d$ and $r n \ln n=\frac{1}{2} p_{1} n(n-1)$ ).

Theorem 2 shows that if $\alpha>\frac{1}{2}$ and $2 e^{-\frac{\alpha}{r}} \geq 1$, then there exists an exact phase transition in the binary case of Model RB. Given the values of $n, d$ and $p_{1}$ in Model $\mathrm{B}$, for the setting of $\left\langle n, d, p_{1}, p_{2}\right\rangle$, the conditions that the equivalent setting in Model RB satisfies Theorem 2 are

$$
\begin{gathered}
\alpha=\frac{\ln d}{\ln n}>\frac{1}{2} \Rightarrow d^{2}>n \\
2 e^{-\frac{\alpha}{r}} \geq 1 \Rightarrow 2 e^{-\frac{\ln d}{\ln n} \cdot \frac{2 \ln n}{p_{1}(n-1)}} \geq 1 \Rightarrow p_{1} \geq \frac{2 \ln d}{(n-1) \ln 2}
\end{gathered}
$$

The proof of Theorem 2 reveals that if the conditions (37), (38) are satisfied, then Model RB will exhibit an exact phase transition at $E(N)=1$. It should be noted that Williams and Hogg (Williams \& Hogg, 1994), and independently Smith (1996) have already developed a theory to predict the phase transition point in Model B on the basis of $E(N)=1$. Prosser (1996) found that this theory is in close agreement with the empirical results, except when $p_{1}$ is small. Inequality (38) shows that in order to make the equivalent setting in Model RB satisfy the conditions of Theorem 2, the parameter $p_{1}$ in Model B should not be less than a certain value, which is consistent with Prosser's experimental finding.

Now we consider a typical setting $\left\langle 20,10,0.5, p_{2}\right\rangle$ of Model B. Let $n=20, \alpha=\frac{\ln 10}{\ln 20} \approx$ 0.7686 and $r=\frac{0.5(20-1)}{2 \ln 20} \approx 1.5856$ in Model RB. Then the setting of Model RB with such values is equivalent to the setting $\left\langle 20,10,0.5, p_{2}\right\rangle$ of Model B. From Inequalities (37) and 
(38) it is also not hard to show that the equivalent setting in Model RB corresponding to the setting $\left\langle 20,10,0.5, p_{2}\right\rangle$ satisfies the conditions of Theorem 2, i.e., $10^{2}>20$ and $p_{1}=$

$0.5 \geq \frac{2 \ln 10}{(20-1) \ln 2} \approx 0.35$. The experiments done by Prosser (1996) show that the instances generated at $p_{2}=0.38$ are very hard to solve. This maximum cost point also agrees well with the asymptotic phase transition point of Model RB that is $p=1-e^{-\frac{\alpha}{r}} \approx 1-e^{-\frac{0.7686}{1.5856}} \approx 0.38$. For some other settings of Model B in the previous work, we can also find their equivalent settings in Model RB using this method. Thus the hardness of solving these settings of Model B is equal to that of solving their equivalent settings in Model RB. From many previous studies (Gent et al., 1999; Smith \& Dyer, 1996; Prosser, 1996) we know that the instances generated at the phase transition in many settings of Model B are very hard to solve for various kinds of CSP algorithms. So there exist a lot of hard instances to solve in Model RB.

\section{Conclusions and Future Work}

A lot of experimental and theoretical studies indicate that a phase transition in solvability is a very important feature of many decision problems in computer science. It is shown that these problems can be characterized by a control parameter in such a way that the space of problem instances is divided into two regions: the under-constrained region where almost all problems have many solutions, and the over-constrained region where almost all problems have no solutions, with a sharp transition between them. Another interesting feature associated with the phase transition is that the peak in hardness of solving the problem instances occurs in the transition region. Since these instances generated in the transition region appear hardest to solve, they are commonly used as a benchmark for algorithms for many NP-complete problems. But unfortunately, except for the Hamiltonian cycle problem (which is NP-complete), all the decision problems that have exact results about the existence and the location of the phase transition are in $\mathrm{P}$ class (Parkes, 1997), e.g. random 2-SAT. These problems are not so interesting as the NP-complete problems from a complexity theoretic point of view because they can be solved in polynomial time. For the Hamiltonian cycle problem, using an improved backtrack algorithm with sophisticated pruning techniques, Vandegriend and Culberson (1998) recently found that the problem instances in the phase transition region are not hard to solve.

In this paper we proposed a new type of random CSP model, Model RB, which is a revision to the standard Model $\mathrm{B}$, and the asymptotic analysis of this model has also been presented. The results are quite surprising. We can not only prove the existence of phase transitions in this model but also know the location of transition points exactly. It was further shown that there exist a lot of hard instances in Model RB by relating its hardness to the standard Model B. Since there is still some lack of studies about the exact derivation of the phase transitions in NP-complete problems, this paper may provide some new insight into this field. However, we did not discuss the scaling behaviour of Model RB and some other related issues in this paper. In order to get a better understanding of Model RB, we suggest that future work should include determining either empirically or theoretically whether or not hard instances persist with reasonably high frequency as the number of variables increases. ${ }^{1}$

1. Two anonymous referees suggest this point. 


\section{Acknowledgments}

This research was supported by National 973 Project of China Grant No. G1999032701. We would like to thank Ian P. Gent, Barbara M. Smith, Peter van Beek and the anonymous referees for their helpful comments and suggestions.

\section{References}

Achlioptas, D., Kirousis, L., Kranakis, E., Krizanc, D., Molloy, M., \& Stamatiou, Y. (1999). Random constraint satisfaction: A more accurate picture. Constraints. submitted. Also in Proc. Third International Conference on Principles and Practice of Constraint Programming (CP 97), Springer-Verlag, pp. 107-120, 1997.

Bollobás, B. (1985). Random Graphs. Academic Press, New York.

Cheeseman, P., Kanefsky, B., \& Taylor, W. (1991). Where the really hard problems are. In Proceedings of IJCAI-91, pp. 331-337.

Dechter, R. (1998). Constraint satisfaction. In MIT Encyclopedia of the Cognitive Sciences (MITECS). Online at “ ftp://ftp.ics.uci.edu/pub/CSP-repository/papers/R68.ps".

Friedgut, E. (1999). Sharp thresholds for graph properties and the k-sat problem. Journal of the American Mathematical Society, 12, 1017-1054.

Frieze, A., \& Suen, S. (1996). Analysis of two simlpe heuristics on a random instance of k-sat. Journal of Algorithms, 53, 469-486.

Frost, D., \& Dechter, R. (1994). In search of the best constraint satisfaction search. In Proceedings of AAAI-94, pp. 301-306.

Gent, I., MacIntyre, E., Prosser, P., Smith, B., \& Walsh, T. (1999). Random constraint satisfaction: Flaws and structure. Constraints. submitted. Online at " http://www.cs.strath.edu.uk/ apes/apesreports.html".

Hogg, T. (1996). Refining the phase transition in combinatorial search. Artificial Intelligence, $81,127-154$.

Kirkpatrick, S., \& Selman, B. (1994). Critical behavior in the satisfiability of random boolean expressions. Science, 264, 1297-1301.

Kirousis, L., Kranakis, E., Krizanc, D., \& Stamatiou, Y. (1998). Approximating the unsatisfiability threshold of random formulas. Random Structures and Algorithms, 12, 253-269.

Larrosa, J., \& Meseguer, P. (1996). Phase transition in max-csp. In Proceedings of ECAI-96, pp. $190-194$.

Mitchell, D., Selman, B., \& Levesque, H. (1992). Hard and easy distributions of sat problems. In Proceedings of AAAI-92, pp. 459-465. 
Parkes, A. (1997). Clustering at the phase transition. In Proceedings of AAAI-97, pp. $340-345$.

Prosser, P. (1996). An empirical study of phase transitions in binary constraint satisfaction problems. Artificial Intelligence, 81, 81-109.

Purdom, P. (1997). Backtracking and random constraint satisfaction. Annals of Mathematics and Artificial Intelligence, 20, 393-410.

Smith, B. (1999). Constructing an Asymptotic Phase Transition in Random Binary Constraint Satisfaction Problems. Extended Abstract.

Smith, B., \& Dyer, M. (1996). Locating the phase transition in binary constraint satisfaction problems. Artificial Intelligence, 81, 155-181.

Vandegriend, B., \& Culberson, J. (1998). The $G_{n, m}$ phase transition is not hard for the hamiltonian cycle problem. Journal of Artificial Intelligence Research, 9, 219-245.

Williams, C., \& Hogg, T. (1994). Exploiting the deep structure of constraint problems. Artificial Intelligence, 70, 73-117. 